\title{
Venusian bow shock as seen by the ASPERA-4 ion instrument on Venus Express
}

\author{
I. Whittaker, ${ }^{1}$ G. Guymer, ${ }^{1}$ M. Grande, ${ }^{1}$ B. Pintér, ${ }^{1}$ S. Barabash, ${ }^{2}$ A. Federov, ${ }^{3}$ \\ C. Mazelle, ${ }^{3}$ J. A. Sauvaud, ${ }^{3}$ R. Lundin, ${ }^{2}$ C. T. Russell, ${ }^{4}$ Y. Futaana, ${ }^{2}$ M. Fränz, ${ }^{5}$ \\ T. L. Zhang, ${ }^{6}$ H. Andersson, ${ }^{2}$ A. Grigoriev, ${ }^{2}$ M. Holmström, ${ }^{2}$ M. Yamauchi, ${ }^{2}$ \\ K. Asamura, ${ }^{7}$ W. Baumjohann, ${ }^{6}$ H. Lammer, ${ }^{6}$ A. J. Coates,${ }^{8}$ D. O. Kataria, ${ }^{8}$ D. R. Linder,${ }^{8}$ \\ C. C. Curtis,${ }^{9}$ K. C. Hsieh,${ }^{9}$ H. E. J. Koskinen, ${ }^{10,11}$ E. Kallio, ${ }^{11}$ P. Riihelä, ${ }^{11}$ W. Schmidt, ${ }^{11}$ \\ J. Kozyra, ${ }^{12}$ S. McKenna-Lawlor, ${ }^{13}$ J. J. Thocaven, ${ }^{3}$ S. Orsini, ${ }^{14}$ R. Cerulli-Irelli, ${ }^{14}$ \\ A. Mura, ${ }^{14}$ M. Milillo, ${ }^{14}$ M. Maggi, ${ }^{14}$ E. Roelof, ${ }^{15}$ P. Brandt, ${ }^{15}$ R. A. Frahm, ${ }^{16}$ \\ J. R. Sharber, ${ }^{16}$ P. Wurz, ${ }^{17}$ and P. Bochsler ${ }^{17}$
}

Received 25 August 2009; revised 2 February 2010; accepted 28 April 2010; published 28 September 2010.

[1] The Analyzer of Space Plasmas and Energetic Atoms (ASPERA-4) instrument on Venus Express is used to determine bow shock position at Venus using ion data alone, using data recorded during a solar minimum from the Ion Mass Analyzer (IMA) which is part of the ASPERA-4 package. Previous models constructed from solar minimum data using Venus Express, Pioneer Venus Orbiter (PVO) and Venera 9 and 10 are also compared to the current fit. An important feature of this new fit is a statistical accuracy introduced in the form of a probability weighting function for the data points, based on the time spent in particular locations. The bow shock curve is then compared to two-dimensional ion maps. These verify the accuracy of this and previous solar minimum fit curves based on PVO and Venus Express magnetic data. Comparing all bow shock models to the 2D ion maps shows that a combination of models produces the best fit. Since all the fitted curves show differences in position they are investigated relative to the solar conditions pertaining at the times when the individual data sets were measured. The sub solar point and terminator distance were thus found to vary linearly with sunspot number and hence with solar activity. This relationship, which was already known to exist between solar maximum and solar minimum, is now shown to exist between different solar minima and even within the same minimum. This indicates a need for the mechanisms for bow shock maintenance and variance to be more closely modeled.

Citation: Whittaker, I., et al. (2010), Venusian bow shock as seen by the ASPERA-4 ion instrument on Venus Express, J. Geophys. Res., 115, A09224, doi:10.1029/2009JA014826.

\section{Introduction}

[2] Without a protective dipolar magnetic field, Venus is open to erosion by the solar wind. This strips away the upper layers of the atmosphere and is thought to have been a cause of the early water loss at Venus [Rasool, 1968]. Although Venus does not have an internal magnetic field, an

\footnotetext{
${ }^{1}$ Aberystwyth University, Penglais, Aberystwyth, Ceredigion, UK.

${ }^{2}$ Swedish Institute of Space Physics, Kiruna, Sweden.

${ }^{3}$ Centre d'Étude Spatiale des Rayonnements, Toulouse, France.

${ }^{4}$ IGPP, University of California, Los Angeles, California, USA.

${ }^{5}$ Max-Planck-Institut für Sonnensystemforschung, Katlenburg-Lindau, Germany.

${ }^{6}$ Space Research Institute, Austrian Academy of Sciences, Graz, Austria.

${ }^{7}$ Institute of Space and Astronautical Science, Sagamihara, Japan.

${ }^{8}$ Mullard Space Science Laboratory, UCL, Dorking, UK.
}

Copyright 2010 by the American Geophysical Union. 0148-0227/10/2009JA014826 electric field is induced due to the motion of the interplanetary magnetic field (IMF) embedded in the solar wind plasma. This in turn leads to draping of magnetic field lines across the planet, which becomes mass loaded. The resulting obstacle leads to the creation of the bow shock boundary [Luhmann et al., 1986]. The inner boundary known as the

\footnotetext{
${ }^{9}$ Department of Physics, University of Arizona, Tucson, Arizona, USA.

${ }^{10}$ Department of Physics, University of Helsinki, Helsinki, Finland.

${ }^{11}$ Finnish Meteorological Institute, Helsinki, Finland.

${ }^{12}$ Space Physics Research Laboratory, University of Michigan, Ann Arbor, Michigan, USA.

${ }^{13}$ Space Technology Ireland, National University of Ireland, Maynooth, Ireland.

${ }^{14}$ Instituto di Fisica dello Spazio Interplanetario, Rome, Italy.

${ }^{15}$ Johns Hopkins University Applied Physics Laboratory, Laurel, Maryland, USA.

${ }^{16}$ Southwest Research Institute, San Antonio, Texas, USA.

${ }^{17}$ University of Bern, Physikalisches Institut, Bern, Switzerland.
} 


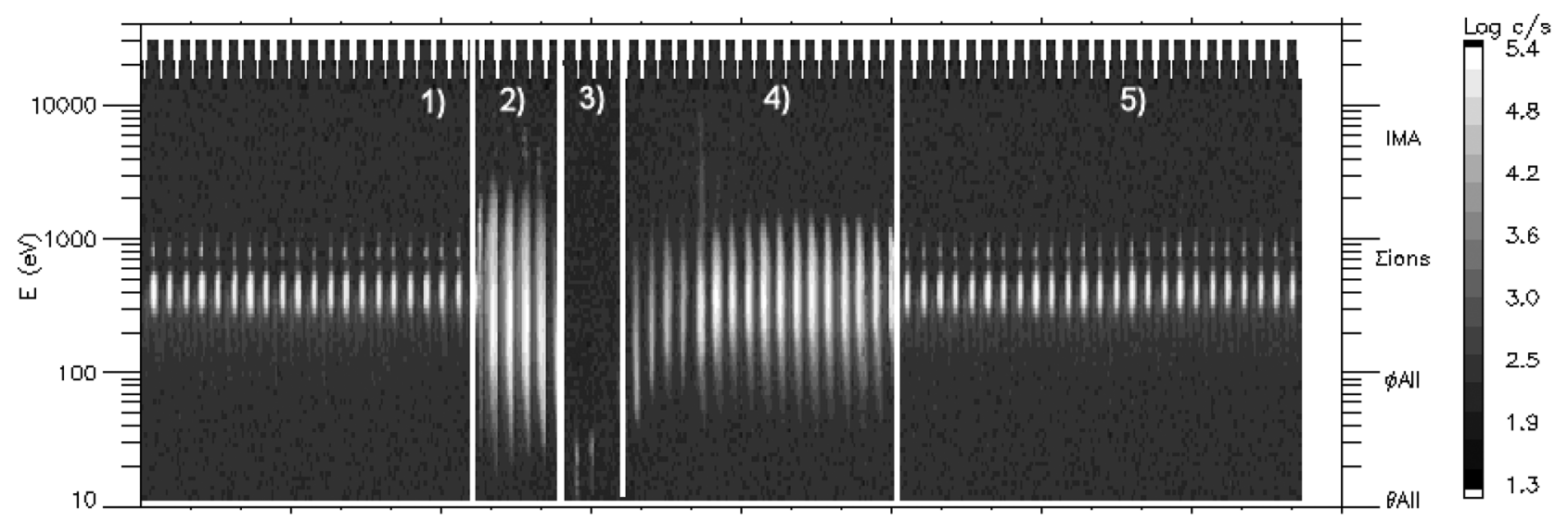

Figure 1. Ion Specrogram observed by the IMA on VEX from the 18th January 2007. Time is shown on the $x$ axis and energy on the $y$ axis. The regions identified are (1) The dayside solar wind, (2) The dayside ionosheath, indicated by the shocking of the solar wind, (3) The inner boundary crossings and ionosphere seen during periapsis, (4) Nightside wake, (5) Exiting the night side bow shock back into the solar wind.

ionopause is defined as the pressure balance between solar wind dynamic pressure and ionospheric thermal pressure. At certain solar minimum conditions it was shown by PVO that the ionosphere could not reach a pressure balance and this resulted in a magnetisation of the ionosphere through downward diffusion and convection [Russell and Vaisberg, 1983; Phillips et al., 1985].

[3] Previous bow shock fits were obtained from the Pioneer Venus Orbiter (PVO) and the Venera spacecraft using magnetic field readings. Magnetic crossing points were used to identify jumps in $|\mathbf{B}|$ when estimating the bow shock position [Russell et al., 1988]. A fit has previously been obtained from Venus Express data using both electron and magnetometer measurements [Martinecz et al., 2008] as well as an entirely magnetic approach that agrees with the Russell model [Zhang et al., 2008]. The position of the bow shock is important as it reflects the amount of mass loading [Russell et al., 1988]. The PVO data were taken over a complete solar cycle allowing comparisons of cycle position. This has been compared already for PVO data and resulted in the shock position above the dawn-dusk terminator increasing from $2.14 R_{v}$ at solar minimum to $2.40 R_{v}$ at solar maximum, where $R_{v}$ is the radius of Venus. The interval discussed here relates to the deepest Solar minimum observed in modern times [Gibson et al., 2009].

\section{Instrumentation}

[4] The ASPERA-4 instrument onboard Venus Express (VEX) is composed of four different sensors: a neutral particle detector (NPD), a neutral particle imager (NPI), an electron spectrometer (ELS) and the Ion Mass Analyzer (IMA). This paper uses only IMA measurements. The instrument is a top hat electrostatic analyzer using variable voltage steps voltages to scan elevation angles and specified ion energies [Barabash et al., 2007]. Thus in each elevation direction it scans through 96 different energy values before changing to the next. A full scan of all elevations and energies takes $192 \mathrm{~s}$. The azimuthal angle can be determined from the particle impact position on the micro channel plates
(MCP). A full description of the instrument is given by Barabash et al. [2007].

\section{Data Collection}

[5] Figure 1 gives an example of recorded ASPERA-4 data. The spectrogram was recorded on 18 January 2007, during a dayside to nightside pass, meaning that the VEX orbit passed closer to local midday before local midnight. The $x$-axis in Figure 1 is time and the $y$-axis is energy, with the gray level of the pixel representing the counts per second. The time resolution for all angles and energies is $192 \mathrm{~s}$. One pixel contains the counts from all 32 mass channels across 16 azimuthal angle increments, for each of the 96 energy steps shown. Sweeping across the eight elevation angles completes the 192-s spectrum. This means that the spectra appear as a series of 'blobs', each split into 8 columns derived from a separate elevation angle. Thus, in the solar wind the changes in elevation show that the middle of the blob is facing toward the ion flow while columns either side are facing away. This produces spectra in a series of look directions creating a rounded shape rather than a simple time series. In Figure 1 we identify figure pertinent features of the orbit crossing.

[6] Region 1 of Figure 1 shows the dayside solar wind upstream of any planetary interaction. The bow shock crossing is clear as an increase in the energy spread of each of the $192 \mathrm{~s}$ spectra, this shocked period is contained within Region 2. The previous 'blobs' extended from $212 \mathrm{eV}$ to $1.106 \mathrm{keV}$ in the solar wind compared to the 'blobs' in the sheath extending from $27 \mathrm{eV}$ to $3.739 \mathrm{keV}$. Giving an energy spread difference of $894 \mathrm{eV}$ to $3.712 \mathrm{keV}$. This change in energy spreads is indicative of crossing a shock region as described by Argo et al. [1967]. Region 3 shows the ionosphere and also periapsis. This region is bounded by the ion composition boundaries (ICB) on either side. The ICBs are in a similar region to the magnetic pile up boundaries to which they are closely linked. Region 4 shows shocked plasma at the rear of the planet, flowing in the wake. Region 5 begins after VEX has passed through the nightside bow shock and gone back into the solar wind. 


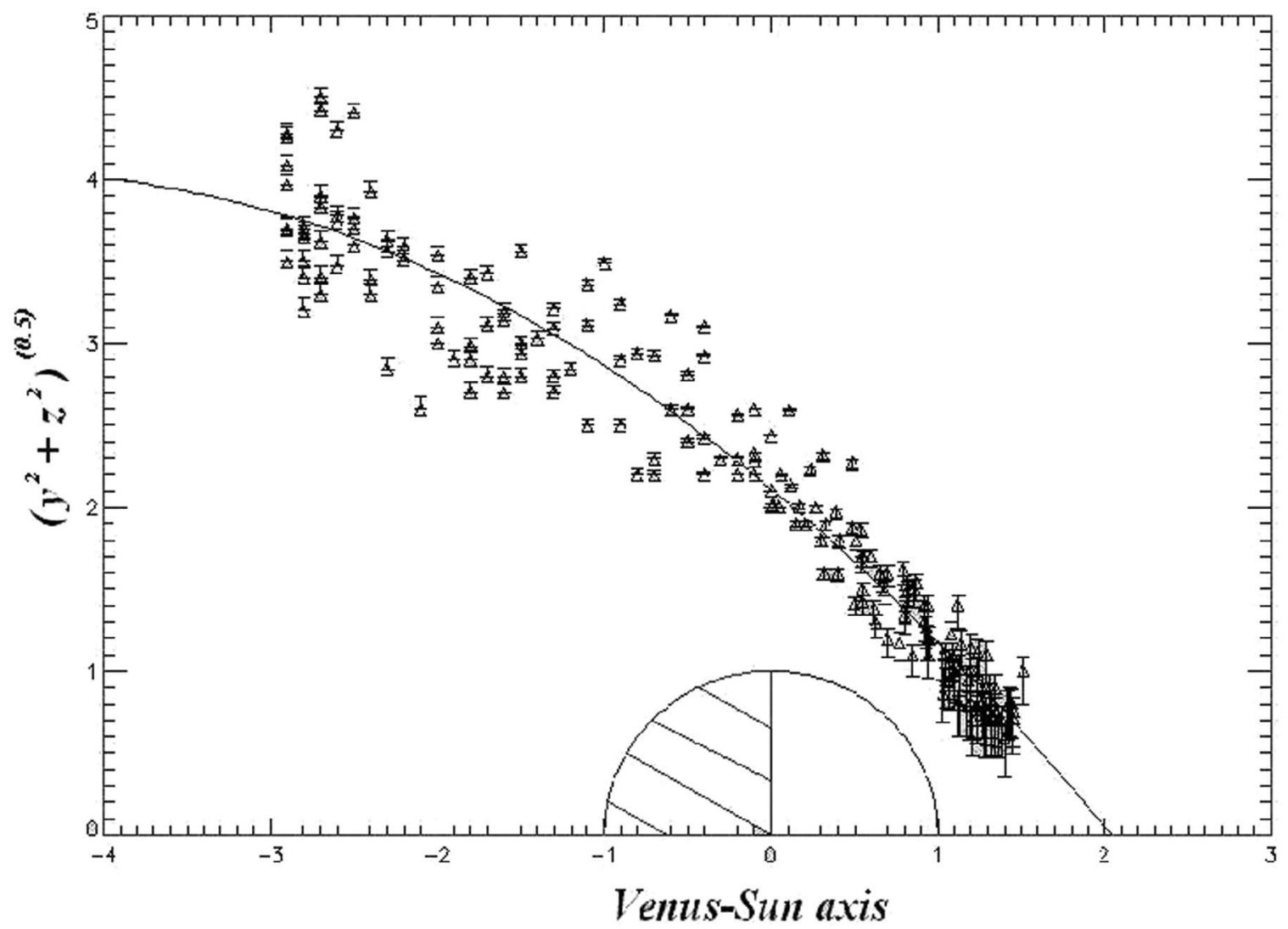

Figure 2. Bow shock crossings inferred from IMA data: the $x$ axis is the Sun-Venus line centered on Venus. The $y$ axis is the distance from this axis using a cylindrical coordinate system. A least squares regression curve is fit to this data. The $x$ axis crossing point can be seen to exceed recognized values for the sub-solar point. The error bars show the maximum and minimum positions that the boundary crossing could be at.

[7] Inbound and outbound crossings were determined for 106 orbits between November 2006 and February 2007. Orbits with missing data or where the crossing point was unclear are omitted. The coordinate system used is Venuscentric and can be seen in Figure 2 projected onto an aberrated solar ecliptic system (assuming a $5^{\circ}$ aberration angle due to solar wind direction). This gives the $x$-axis as the line from the center of Venus to the Sun. The $z$-axis is positive in the direction of the north pole of the planet and the $y$-axis is tangential to the orbital path of the planet, thereby completing the right hand system. All coordinate systems are in units of Venus radii $\left(1 R_{v}=6051.8 \mathrm{~km}\right)$. Data are represented in cylindrical coordinates in a plot of $x$ against $r$, where;

$$
r=\left(y^{2}+z^{2}\right)^{1 / 2}
$$

The crossings can be seen in Figure 2, which show each crossing as a triangle with an error bar. It should be noted that the closer to the planetary surface the crossing point is the greater the associated error bar. This is due to Venus Express having its orbital periapsis point in the northern polar region. This is the point at which it is traveling fastest and therefore the positional error is largest.
[8] The fitting of a curve to the bow shock data in Figure 2 was initially done using a least squares regression algorithm. This was done to check the accuracy of a straight fit with no weighting factors. The easiest parameter with which to make comparisons is the sub-solar point of the bow shock, which gives an indication of the compression of the shock area.

[9] Figure 2 shows a sub-solar crossing point at 2.11 Venusian radii $\left(R_{v}\right)$, much further than previous models. An explanation for this is the importance of positional accuracy as illustrated by the error bars which reflect a temporal resolution of $192 \mathrm{~s}$. This is significant when the spacecraft is at periapsis and traveling close to $10 \mathrm{~km}^{-1}$. The spatial probability distribution described in the next section is introduced to compensate for this by adding a weighting factor.

[10] Figure 3 shows a midday-midnight cut showing the locus of the closest approach of the orbit of Venus. It is clear that the low spatial resolution at periapsis velocity, combined with sampling biases introduced by the minimum altitude that VEX can observe, is leading to a distorted best fit value for the subsolar point, which is never directly sampled. Thus a spatial probability distribution must be used to weight the data points. Similar versions of this method have been used at the Earth [Formisano, 1979] and at Saturn [Masters et al., 2008]. Figure 3 also contains the 


\section{Venus Boundary Crossings}

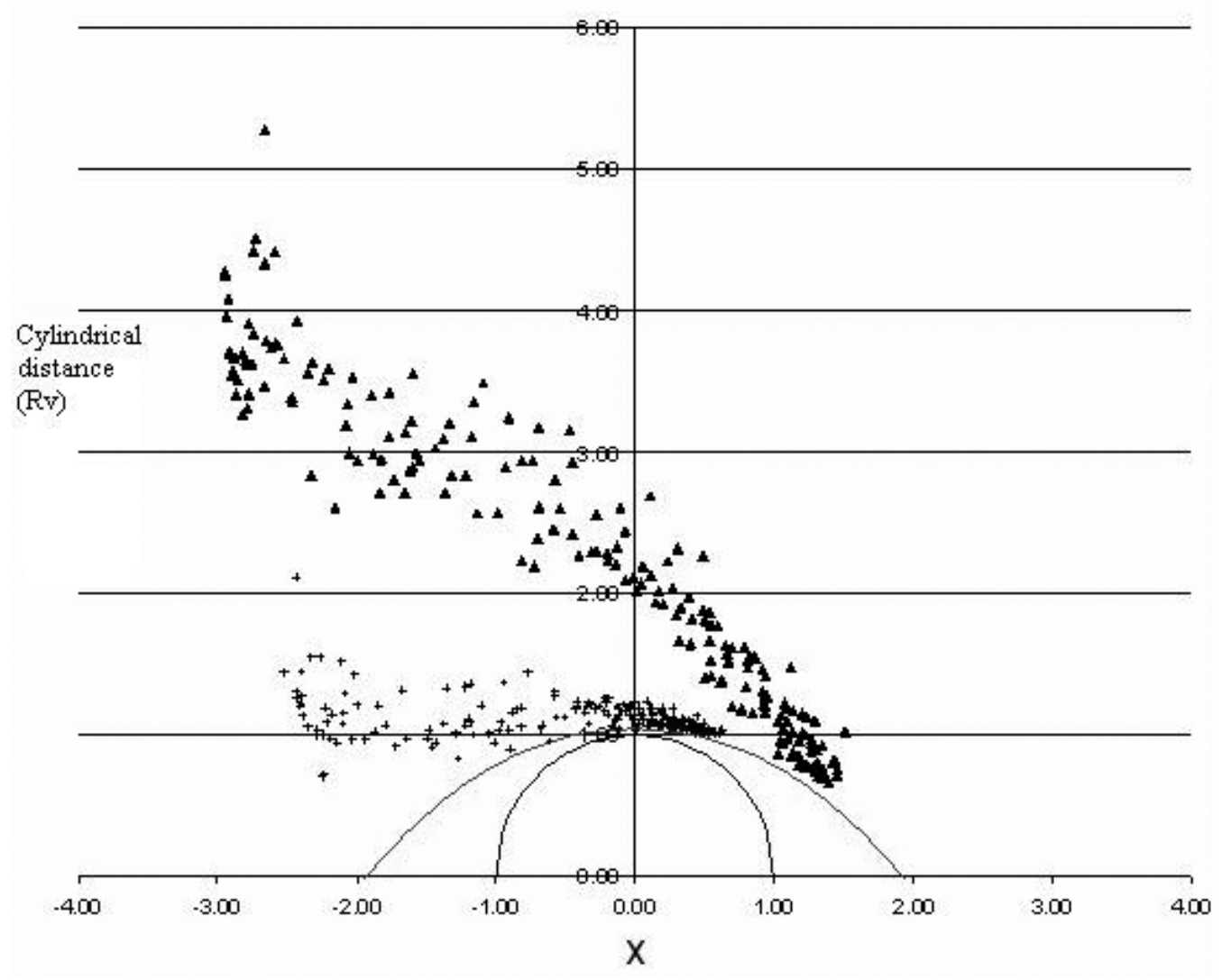

Figure 3. The curve shows the minimum altitude that Venus Express reaches in the cylindrical coordinate system. Evidently no data can be taken lower than this and results in selection effects close to the curve. The triangles are bow shock crossings and plus signs are the inner boundary crossings.

ICB boundary represented by plus signs, where it can be seen that the same selection effects occur.

\section{Spatial Probability Distribution}

[11] The spatial probability distribution (SPD) is found through the use of orbital mechanics. Figure 4 shows all the orbital parameters including the mean $(M)$ and true $(\theta)$ anomaly. These are angles that are related to the position of the spacecraft in its orbit. The true anomaly is the angle from the periapsis point to the current position in the orbit with the orbited body forming the focus point. The mean anomaly is an angle that changes linearly with time. An auxiliary circle is included in Figure 4 and is a circle with two of its points at the periapsis and apoapsis points of the elliptical orbit. Hence it has a radius equal to the semi-major axis of the ellipse. This means that as the orbit is circular it changes linearly with time making the mathematics simple to apply. The eccentric anomaly $(E)$ is the angle from periapsis to the current position on the auxiliary circle and is an intermediate step between the true anomaly and the mean anomaly.

[12] By setting up a grid of positions where VEX could be and determining the amount of time it takes to cross each cell, the probability of occupation can be determined. Figure $5 \mathrm{a}$ represents the process and shows a quarter of the orbital ellipse. This allows all points to be covered, as no bow shock crossings have been detected further than $6 R_{v}$ from the planet. At each cell wall the position on the auxiliary circle of the orbit is found. The time difference can then be derived between when it crosses one wall and the next. Initially the grid is set up by finding the size of the orbit semi-major and semi-minor axes.

[13] The initial set-up variables were taken from the positional data; each orbit takes $23 \mathrm{~h}$ and $59 \mathrm{~min}$ and the point of closest approach is at $0.04 R_{v}$ altitude. By using orbital mechanics equations exact values of the other orbital parameters are obtained. These can then be used to set up a program to find the eccentric anomaly at any point given $x, y$ and $z$.

$$
\begin{gathered}
E=a \cdot \cos \left[\left(1-\frac{\bar{r}}{a}\right) e^{-1}\right] \\
M=E-e \sin E
\end{gathered}
$$

From which

$$
d M=\left\langle E_{1}-e \sin E_{1}\right\rangle-\left\langle E_{2}-e \sin E_{2}\right\rangle
$$

where $E$ is eccentric anomaly

$M$ is mean anomaly 


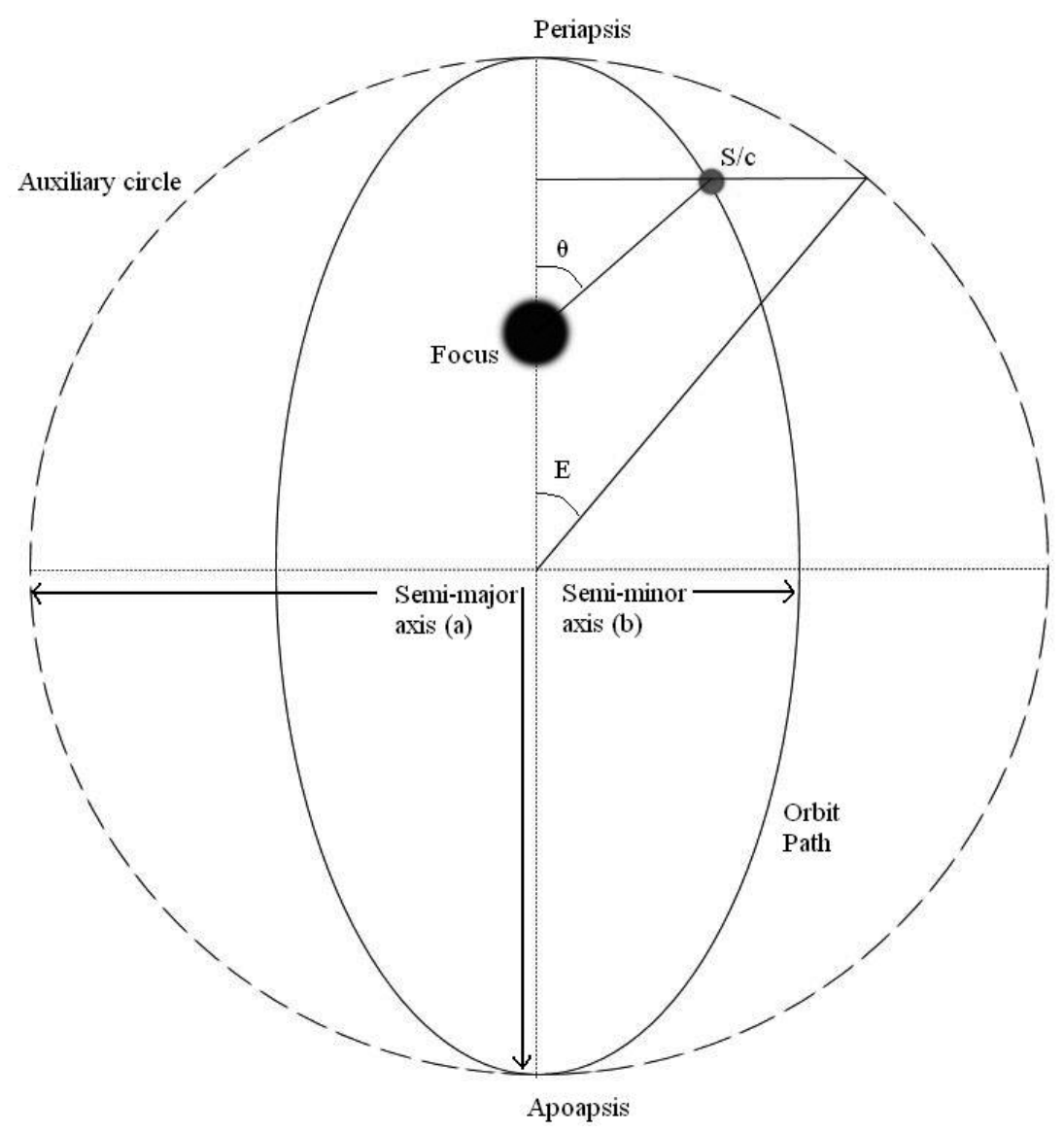

Figure 4. Orbital mechanics illustration. The solid line ellipse is the orbital path, the dashed circle is known as the auxiliary circle and has a radius equal to the semi-major axis of the ellipse. The focus is the planet being orbited and $\mathrm{S} / \mathrm{c}$ is the spacecraft. Angle $\theta$ is the true anomaly and $\mathrm{E}$ the eccentric anomaly.

$e$ is the eccentricity of the orbit

$a$ is the semi-major axis of the orbit

$r$ is the distance from the focus (Venus) to the point on the ellipse in question.

[14] From equation (4) the difference in mean anomaly can be obtained, leading to the time difference, which is the total time multiplied by the mean anomaly difference. Once this is known its value can be assigned to the appropriate grid square. For a midday midnight orbit there is no change in the $y$ direction simplifying the analysis. This makes $r$ in equation (2) the resultant distance of the $x$ and $z$ components only. A bow shock crossing can then be assigned an accuracy which depends on the particular grid square into which it falls.

[15] The initial times of moving into a grid square and leaving it are noted. The time difference over the total time as a property of the grid square then becomes a value for the square. Since the grid size is adjustable, this means that the accuracy can be increased by utilizing more computing power. The output of the program obtained with a cell size of one planetary radius is shown in Figure $5 \mathrm{~b}$. The shade of each cell gives the fraction of the total time of the orbit. Figure $5 \mathrm{~b}$ shows that the most time spent is in the cell at the bottom right hand corner. This is expected as it is the furthest cell from periapsis in the grid. The shade gets darker toward periapsis, indicating that less time is spent in each cell as the velocity increases. The position of VEX is sometimes shared between two cells causing both to be darker than the following cell. However, totaling each row will result in the correct amount of time. Figure 5c shows the SPD grid using a cell wall size of $0.001 R_{v}(\sim 6 \mathrm{~km})$. With an orbital precession rate of $1.6^{\circ}, 56$ orbital paths of VEX are depicted as the orbit rotates from a midday-midnight to a dawn-dusk terminator orbit. This is included for completeness. The next section details a simpler method for handling bow shock crossings that are not on the terminator orbit.

\section{Fitting a Curve to Bow Shock Points}

[16] The original SPD grid applies only to a terminator orbit and as the bow shock data was taken from many different orbits, they cannot be directly compared. $x$ and $r$ bow shock values are combined to create an altitude value $S_{\text {bow }}$. This is then compared with the altitude probability for the terminator orbit which is constant. All the values are weighted depending on the fractional occupancy of the appropriate cell.

$$
S_{\text {bow }}=\left[x_{\text {bow }}^{2}+y_{\text {bow }}^{2}+z_{\text {bow }}^{2}\right]^{1 / 2}
$$




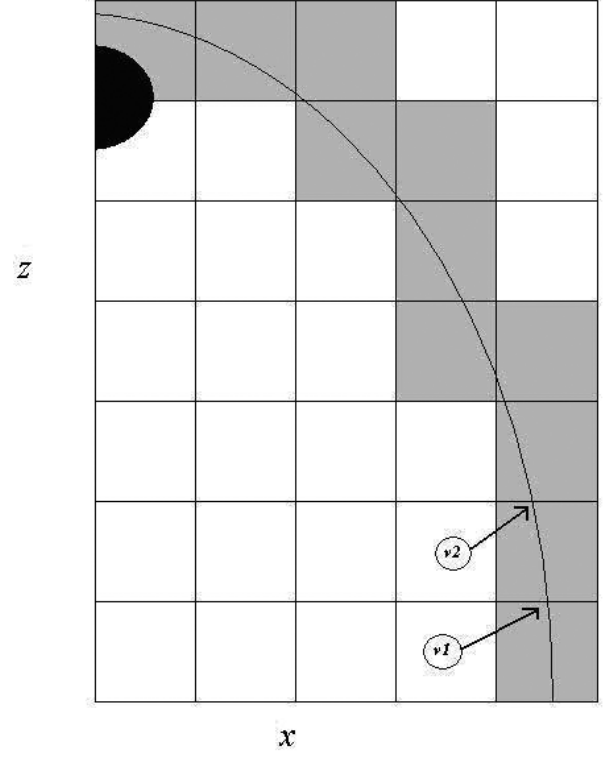

a)

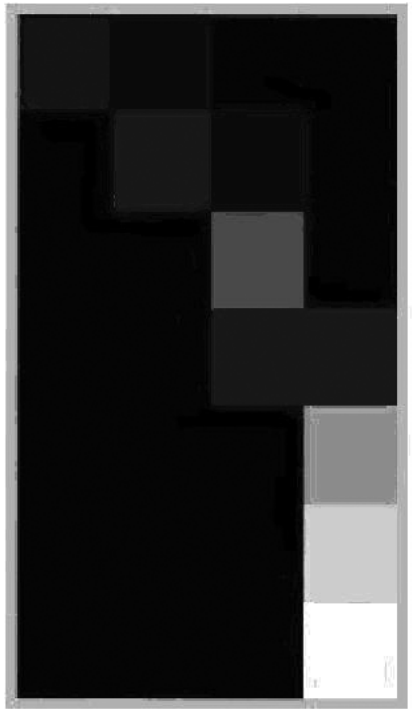

1 Rv grid size

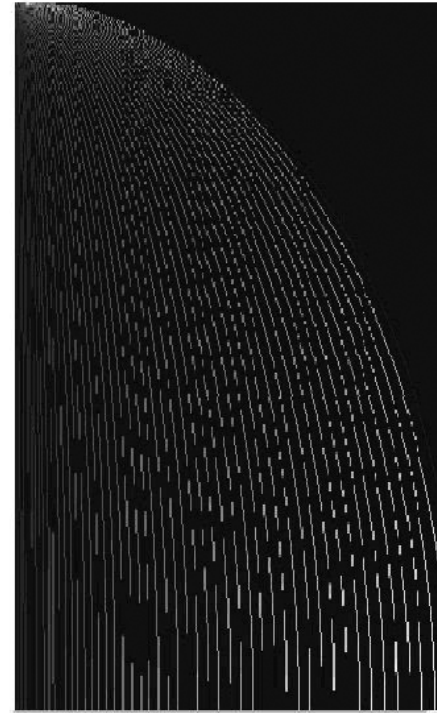

0.001 Rv grid size

b)

Figure 5. (a) A schematic of how the program works. The grid is set up according to the users requirements; the black semi-circle represents the position of Venus. At each cell boundary the position of the orbit and then the eccentric anomaly is found. From this the time difference in each cell is found. (b) This is an actual output from the program. The cell size is one Venusian radius $(6051 \mathrm{~km})$. The color of each cell represents the time spent by Venus express in each, the brighter the square the more time spent. (c) This grid has a cell size of $6 \mathrm{~km}$ and also covers 56 orbits as it rotates from the terminator.

$S_{\text {bow }}$ is the distance from the focus (Venus) to the point on the orbit in question

$x, y$ and $z$ are all position components in a Venus-centric coordinate system.
[17] For each $S_{\text {bow }}$ value the true anomaly value, $T$, is found by:

$$
T=\cos ^{-1}\left\{\left[\frac{a\left(1-e^{2}\right)}{S_{\text {bow }}}-1\right] \times \frac{1}{e}\right\}
$$

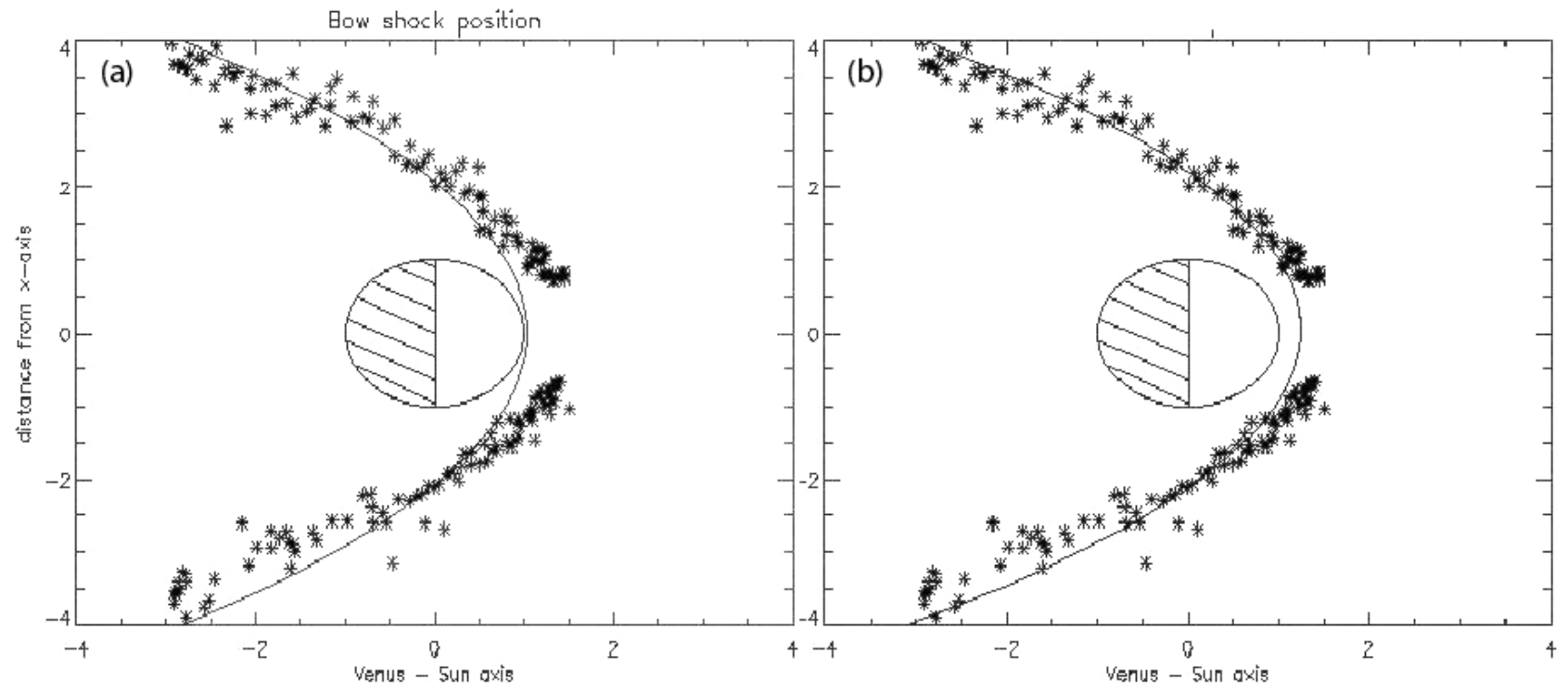

Figure 6. (a) The bow shock data fitted with a curve, the weighting being done with a grid size of one planetary radius $(6051 \mathrm{~km})$. The sub solar point occurs almost on the surface of the planet. (b) By decreasing the grid size the accuracy is raised and the sub solar point reaches an asymptotic value. This is shown here with a grid size of $6 \mathrm{~km}$. 


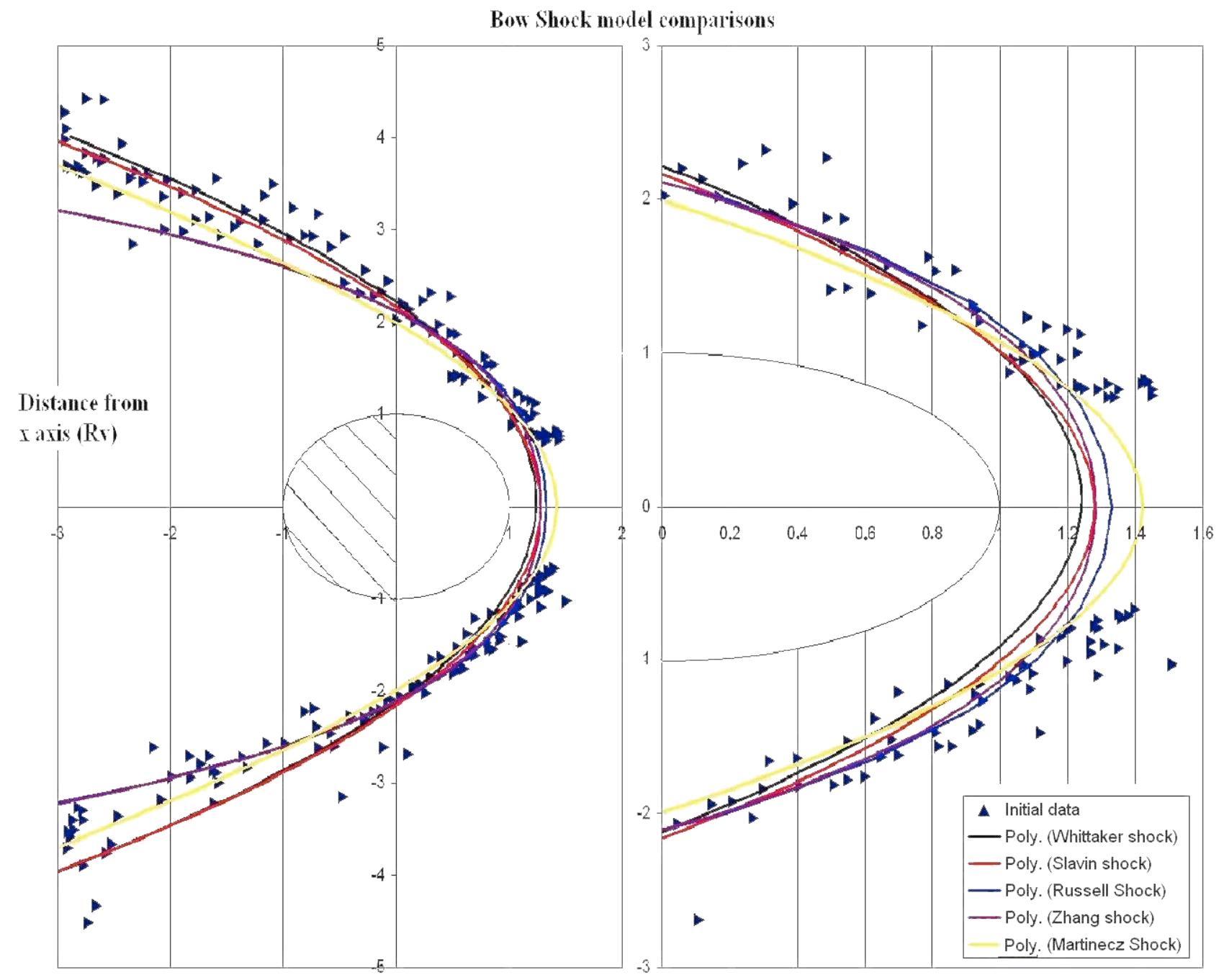

Figure 7. A comparison of the curves used by previous papers. As can be seen, the curve in this paper has a sub solar point closer than any other model so far at solar minimum.

To accurately weight each point a new data array is created with each point counted a number of times proportional to its SPD. This gives nearly all values many entries into the new array, ensuring that all points are counted while still maintaining their weighting.

[18] The new curve is fitted and, as mentioned previously, depends upon the computed grid size. The difference in results on changing the grid size is large enough to make an appreciable difference. Figure $6 \mathrm{a}$ shows a cylindrical plot with a cell wall size of 1 planetary radius and this returns a sub-solar point of $1.03 R_{v}$.

[19] On reducing the size of the cells but increasing their overall number, the value of the sub-solar point moves out along the $x$ axis. This continues until the value of the subsolar point settles toward an asymptotic value of $1.24 R_{v}$. This is shown in Figure $6 \mathrm{~b}$ which has a box size of $0.001 R_{v}$ $(\sim 6 \mathrm{~km})$.

[20] The Bow Shock equation is thus;

$$
x=-0.2641 r^{2}+0.0246 r+1.2399
$$

It should be noted at this point that due to the direct curve fitting on the weighted points it has resulted in an asymmetry in $r$. As the value of $r$ is negligible compared the $r^{2}$ term it can effectively be ignored to produce:

$$
x=-0.2641 r^{2}+1.2399
$$

\section{Comparison With Other Curves}

[21] The present Bow Shock model is compared with four other fits taken during solar minima, as a comparison at a different solar phase would result in a different shape and distance. Comparisons of the models are shown in Figure 7 and are summarized below. The Zhang et al. [2008] fit is not included in the graph as it lies too close to the Russell et al. [1988] curve to be seen correctly.

[22] Information arranged as author, ssp, and line color: current paper, 1.2398, Black; Slavin et al. [1984], 1.277, Red; Russell et al. [1988], 1.33, Blue; Zhang et al. [1990], 1.283, Purple; Zhang et al. [2008], 1.32, n/a; and Martinecz et al. [2008], 1.422, Green. 


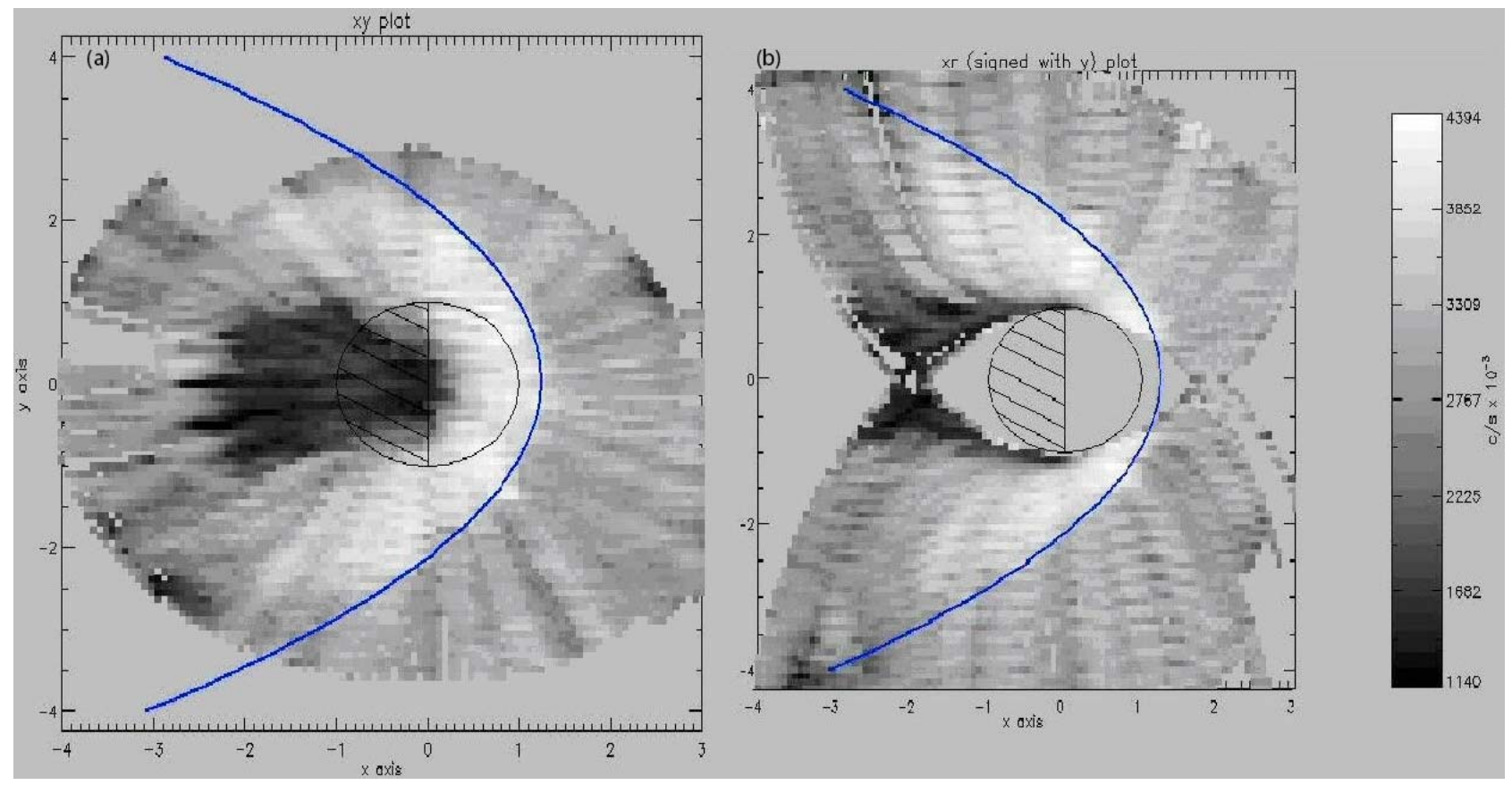

Figure 8. Two ion maps created by binning and averaging 7 months of IMA data into $2 \mathrm{D}$ positions. The gray scale of the pixels represents the average count rate for that area. The scale is in count rates due to uncertainties in the aperture calibration factor. The calculated bow shock has been plotted over these as the blue curve. (a) The map in an $x-y$ coordinate scheme. (b) In cylindrical coordinates with $x$ against distance from $x$ axis signed with $\mathrm{y}$.

[23] Figure 7 includes two diagrams; both are in cylindrical coordinates with $x$ plotted against $r$. The second diagram is a close-up of the first, focusing upon the sub-solar point distance and only looking at $x>0$. All the fits can be seen to pass close to a large number of the data points; the only curve that passes through a reasonable number of data points near the sub-solar point is by Martinecz et al. [2008]. As this model also uses IMA data it is entirely possible that the altitude sampling effect made an impact on the sub-solar point distance. However, as previously discussed these particular points incorporate such a large error that the statistical accuracy needs to be considered.

[24] The main differences between the models are the distance of the sub-solar point from the planetary surface and the shape of the curve. It can be seen that the model described in this paper has the closest sub-solar point. The other models use a conic-section to fit the points and employ variables which include a semi-latus rectum. This is the length of the line from the focus to the bow shock perpendicular to the $x$-axis and involves use of the eccentricity of the curve.

[25] Although a similar approach could have been taken with the present data, a simple parabola produces a perfectly acceptable way to describe the bow shock. With other fits already available it is best to test the fit of this curve using the ion data sets to ensure its accuracy.

\section{Ion Distribution}

[26] To test the current bow shock fit, it is superimposed upon real data and the resulting fit examined. The ion dis- tribution maps shown in Figure 7 comprise seven months of data from January to July in 2007. Seven months of data were used as that is how long it takes for Venus Express to sample all $x$ and $y$ values.

[27] The count rates of all spectra recorded during every orbit are stored and binned within a grid system. For each orbit, values were added to the cells which are passed over. Each box in the grid was then averaged with respect to all the values placed within it. The results were next smoothed and maps created for individual species and plot types.

[28] The maps were made with $x$-values between $3 R_{v}$ and $-4 R_{v}$ and $y$-values between $-4 R_{v}$ to $4 R_{v}$. These were then filled using a bin packing algorithm creating a mean value for each bin. Bins closest to the origin had greater accuracy as more orbits passed through these points thus providing more values for the averaging. Bins near the edges of the map may only have a single orbital pass and were hence less accurate.

[29] Figure 8a shows a map for hydrogen ions in the $x-y$ plane, this is essentially a view looking down on the pole. Figure $8 \mathrm{~b}$ is a cylindrical plot but the $r$-value is signed with $y$ (i.e., $r$ is positive if $y$ is positive and negative if $y$ is negative). Figures $8 \mathrm{a}$ and $8 \mathrm{~b}$ both display bow shock plots and show that the model almost perfectly separates into two regions of ion density. It would be almost impossible to cover all the pixels accurately as the bow shock is not completely stationary and also foreshock effects can create an increase in counts on the ion maps. With the width of one pixel equal to $0.08 R_{v}$ and the bow shock being mostly within the same pixel the error in the bow shock is going to be $\pm 0.04 R_{v}$. At the sub-solar point this gives an error of $3 \%$, 

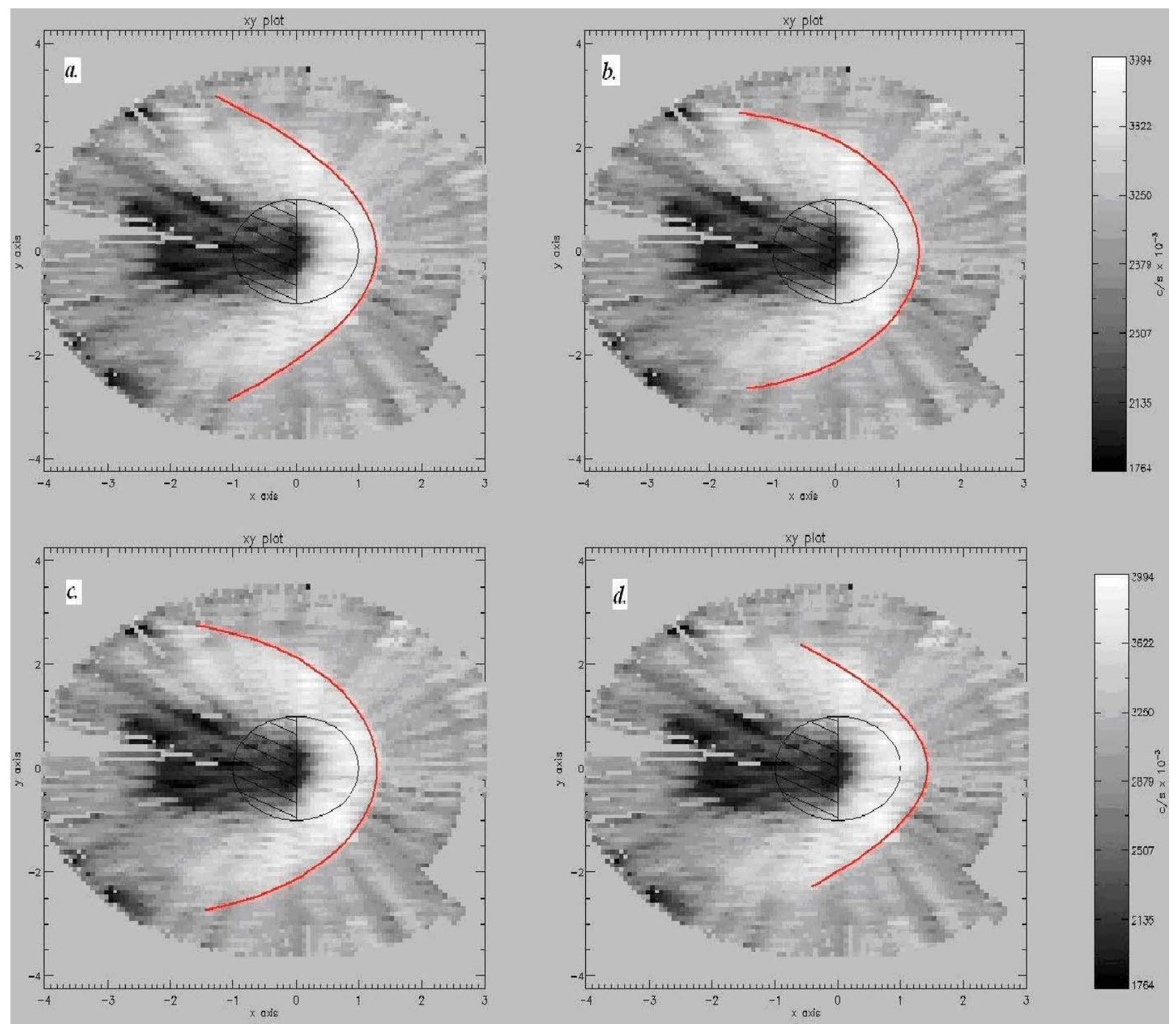

Figure 9. A comparison of the other models plotted on top of the ion maps. (a) The Slavin model. (b) The Russell model. (c) The Zhang model and (d) the Martinecz model.

with the sub-solar point being the closest part of the model. This means that the error decreases with distance.

\section{Analysis}

[30] Investigation of the bow shocks on the maps shows that the fit is accurate for the current Venus Express data. The justification for this is that where the bow shock model is plotted the number of cells from the high density edge is at a minimum. This suggests that the unique weighting method of the data points described above provides an accurate bow shock model. By including more data the bow shock equations could be computed for a set period of time and compared with the next set to see if movement of the bow shock can be determined. This study was performed by inspecting each data set by hand to find bow shock crossings. If an algorithm to determine the crossings automatically were to be created a more extensive investigation could be conducted.
[31] To make further comparisons here the other bow shock fits were plotted onto the ion maps. Figure 9 shows each fit, from this it can be seen that all the curves are in close agreement around the dayside of the planet. However, past the dawn-dusk terminator only the fits of Russell et al. [1988] and Zhang et al. [1990] still hold accuracy. This suggests that for daytime purposes all the fitted curves offer an approximately equal response.

[32] Within the nightside bow shock, the response is best described by a Mach cone [Slavin et al., 1984]. In an approach used by Trotignon et al. [2006], two conic sections were fitted instead of one, which resulted in a better description of the far nightside tail at Mars. In a similar manner Zhang et al. [2008] used a cone angle of $10.5^{\circ}$ to model the nightside area of the bow shock at Venus, with the cone taking over where it intersected the conic section. Using a similar principle for this model, the location of overlap between the two curves occurs at $94^{\circ}$ rather than the 


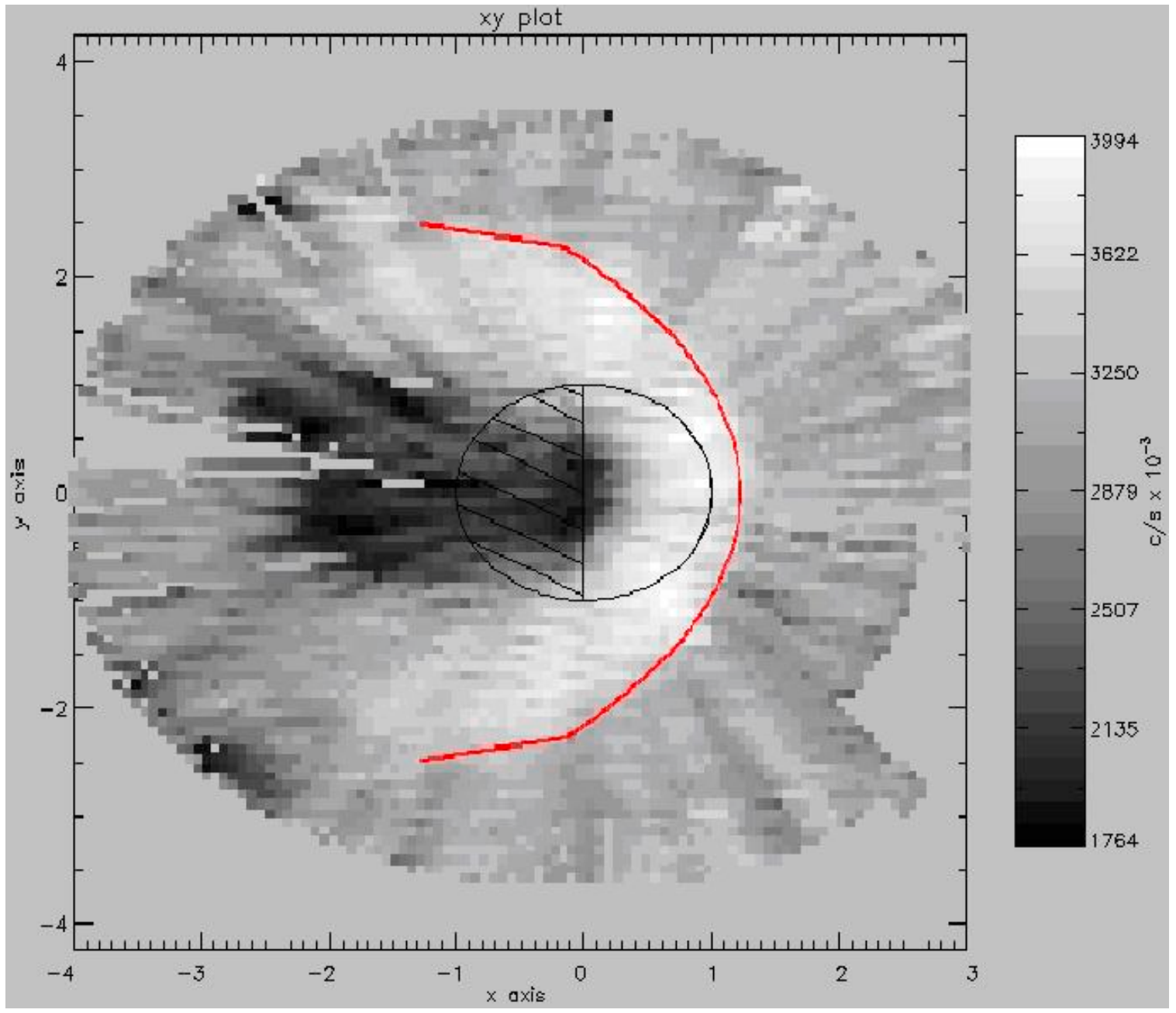

Figure 10. The mixed model, this figure shows a combination of this paper's model and a mach cone of 10.5 degrees. This nicely encompasses the large count density sections in the ion map.

$117^{\circ}$ angle due to the shape of the fit. This final model is now the current standard to be used in investigations of the bow shock region. This is shown in Figure 10 and has a form of;

For $x \geq-0.1647$

$$
x=-0.2641 r^{2}+0.0246 r+1.2399
$$

For $x<-0.1647$

$$
s=\frac{2.26}{\sin (\theta+10.5)}
$$

where $s$ is the distance from the $x$-axis $s=\left(y^{2}+z^{2}\right)^{1 / 2}$ and $\theta$ is the angle from the $x$-axis $\theta=\tan ^{-1}\left(\frac{s}{x}\right)$.

[33] Also of interest are anomalies with respect to the curved shape of the data in the ion maps. Modeling of the foreshock at Venus with respect to bow shock or solar wind conditions and position would allow the ion maps to be cleaned up to show only features important to the study at the time. The ion maps are of high importance and further work in this regard is going into the investigation of solar wind activity, as well as the distribution and paths of both solar and planetary origin ions. Ion maps could in addition help to identify long-term trends by showing the build up and average counts particular to different species.

\section{Comparing Solar Conditions to Each Bow Shock Fit}

[34] It is not surprising that the fitted curves for each case are slightly different as they come from different solar minima and different time periods within these minima. The differences between the fitted bow shock curves could arise from either the data type used to derive the bow shock position or the solar conditions at the time. If the data type were the cause then it would result in a description that the magnetic bow shock is in a different location to the plasma bow shock. This doesn't seem reasonable however as the frozen-in flow should result in the bow shocks being in approximately the same location.

[35] This leaves changes in solar conditions to be the factor in the position and compression of the bow shock locations. To investigate this further the time periods of each curve are found and then the sunspot number and F10.7 index (as an indicator of Extreme UltraViolet (EUV) flux) 

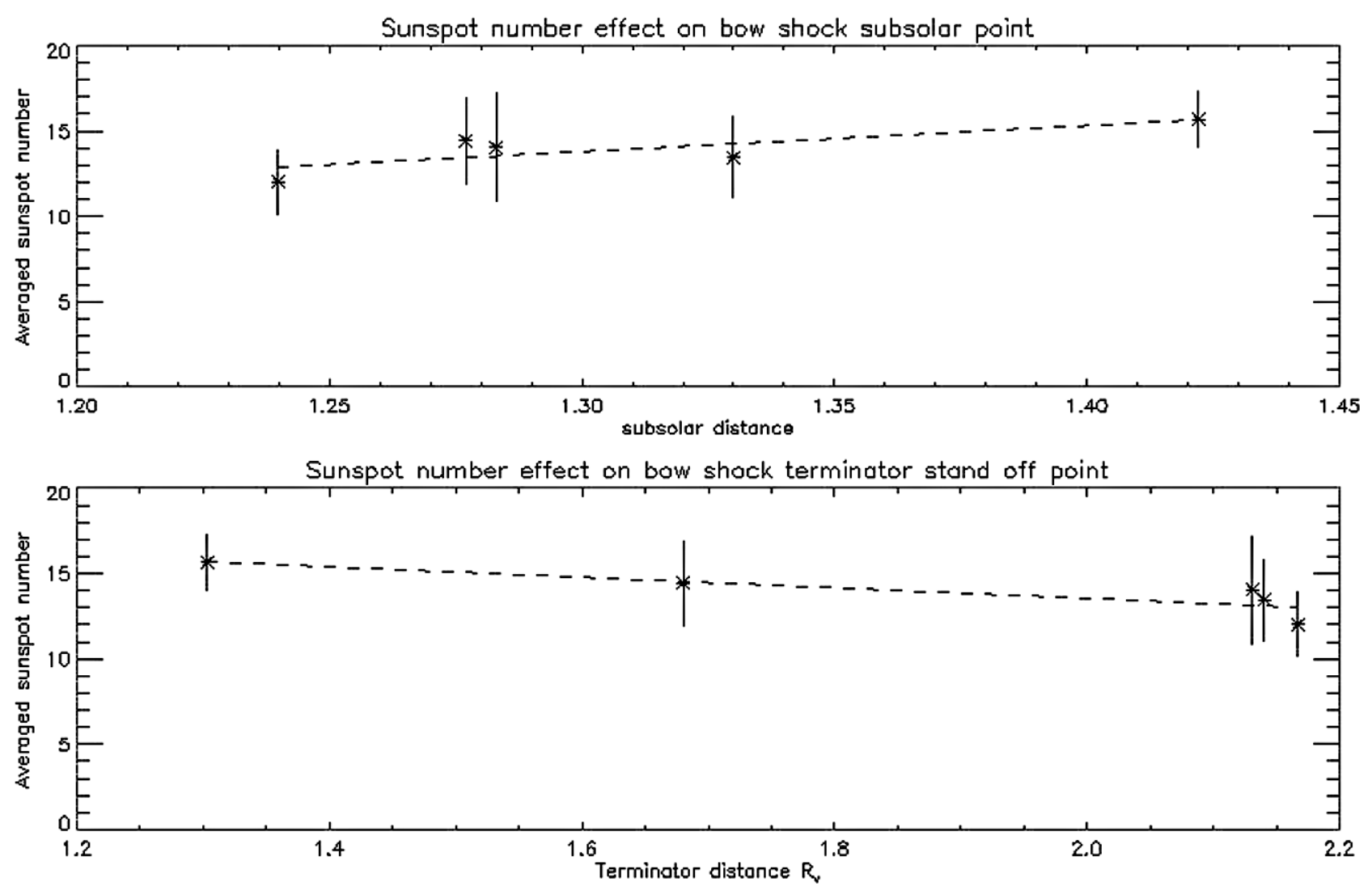

Figure 11. The averaged sunspot number for each period is plotted against (top) the subsolar distance and (bottom) terminator stand off distance of each bow shock fit. The error bars correspond to a single standard deviation either side of the data point for that time period. The fit line passes through all data point error bars in both cases. We can therefore conclude that the solar activity (using the sunspot number as a proxy) has a linear relation to the bow shock shape and size.

are compared to both the sub solar point and the terminator distance to the bow shock. The terminator distance is the same as the semi-latus rectum in the three point curve fit and is referred to as $L$ in the equations using this type of fit.

[36] The data from this paper and from Martinecz et al. [2008] came from two different time periods between May 2006 and February 2007 using VEX. The Russell et al. [1988] and Zhang et al. [1990] curves are based on earlier PVO data, using data from May to September 1986 period and from season 13 of the data set (September 1986 to November 1986), respectively. Acquiring the solar data for the Slavin et al. [1984] curve is more problematic as there are multiple instruments used over a wide ranging time period.

[37] Slavin et al. [1984, p. 2709] stated, "The lower inclination and less eccentric nature of their orbits make the Venera 9 and 10 shock crossings better suited for modeling the Venus shock." Therefore the data taken from the Venera crossings are assumed to be the most important ones and the solar conditions at this point are counted, (October 1975 to April 1976).

[38] The F10.7 data has been taken from the National Geophysical Data Center (NGDC), part of the National Oceanic and Atmospheric Administration (ftp://ftp.ngdc. noaa.gov/STP/SOLAR_DATA/SOLAR_RADIO/FLUX/ Penticton_Observed/2007/2007.OBS). The Lyman alpha data has been taken from the data set at the Laboratory for Atmospheric and Space Physics, University of Colorado [Woods et al., 2000].

[39] Information organized as author, average sunspot number, average F10.7 flux: current paper, 12.025, 83.0992; Slavin et al. [1984], 14.429, 75.4966; Russell et al. [1988], 13.46, 69.6352; Zhang et al. [1990], 14.05, 80.0689; Martinecz et al. [2008], 15.683, 77.3161.

[40] Figure 11 shows the sunspot number plotted against the variables of each bow shock fit. Figure 11 includes error bars for each time period that give an indication of the spread of solar conditions as each error bar is one standard deviation in height.

[41] Although there are only five points there is still a strong linear relationship between sunspot number and both the sub-solar point and terminator distance. The fit line passes through all the error bars on the graphs and shows that there is still variation with solar activity at solar minimum. The increase in sunspot number corresponds to a decrease in terminator stand off distance as previously seen in PVO data [Phillips and McComas, 1991]. However, the sub-solar point increases showing that the shape of the bow shock changes with sunspot number.

[42] The actual cause of this movement of the bow shock was investigated by looking at proxies of the two emitted frequencies that excite $\mathrm{H}$ and $\mathrm{O}$. These are the F10.7 index 


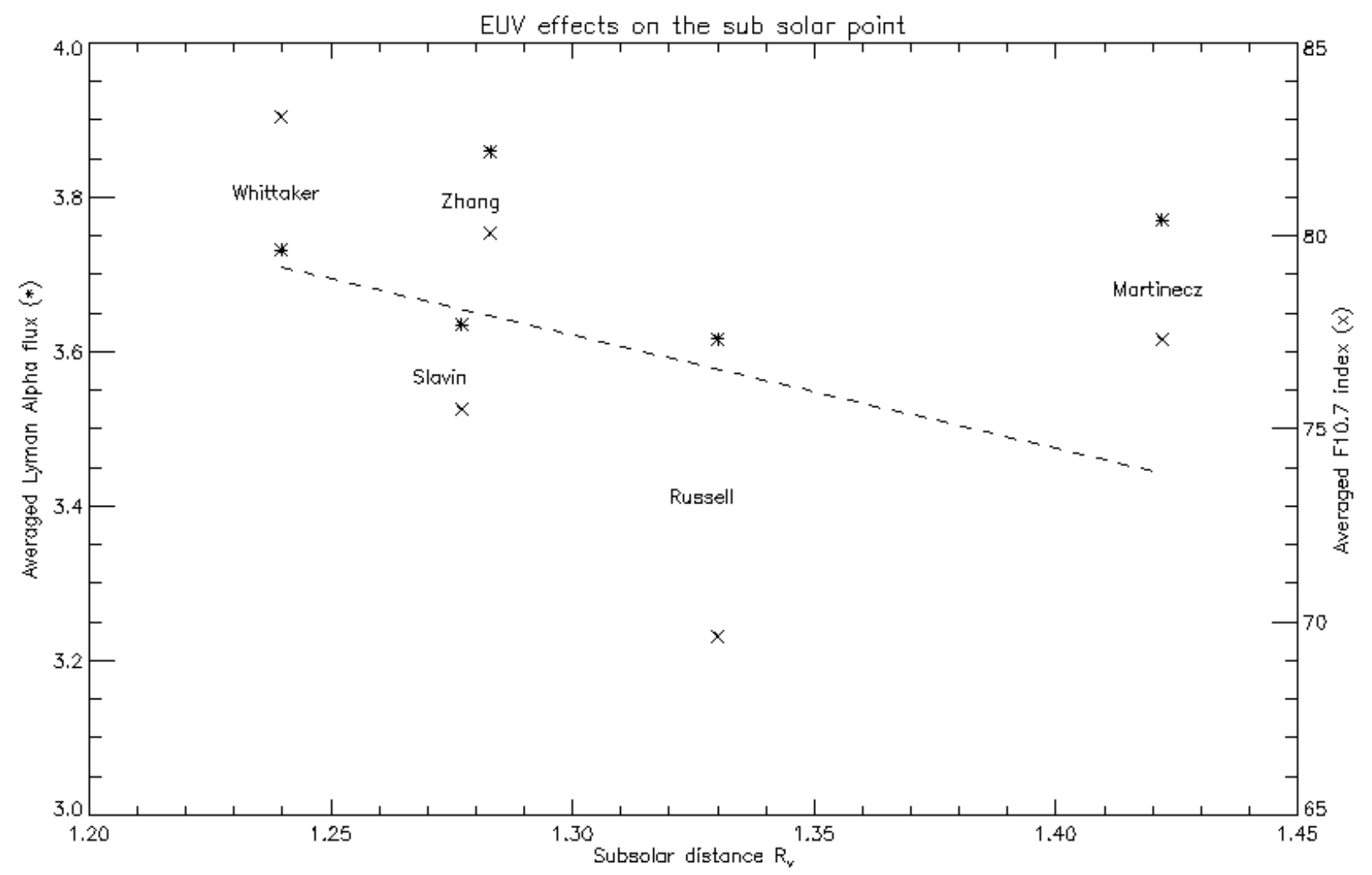

Figure 12. This plot shows both the averaged F10.7 data values (cross) and averaged Lyman alpha flux (asterisk) for each time period. The Lyman alpha data points seem to suggest that the flux has very little effect on the bow shock as the fit line is almost parallel to the $x$ axis. The dashed fit line shown is a linear regression of all five F10.7 data points. The line does not fit the data points as well as the previous determination.

which is an indicator of solar EUV emission, and LymanAlpha radiation which ionizes hydrogen atoms. The plot comparing terminator stand off distance to the EUV and Lyman alpha shows no correlation and is not included in this discussion.

[43] Figure 12 shows the Lyman Alpha and F10.7 index with respect to the sub-solar position. These data points are not as correlated to position as the sunspot numbers are. Looking at the Lyman alpha flux initially, the star $(*)$ data points in Figure 12, they do not show any trend at all and the fit line is almost parallel to the $x$-axis. This provides a strong indication that the Lyman alpha flux has very little effect on the bow shock position.

[44] The F10.7 index however presents a closer relationship. The dashed line in Figure 12 shows the linear regression line fitted to these data. The data points do not lie exactly on the fit line but they do show a negative trend with sub-solar point. This suggests that although the EUV is important, there is evidently another factor involved.

\section{Conclusions}

[45] By introducing statistical accuracy to a number of bow shock crossings a new bow shock fit has been produced to accurately model the Venusian bow shock position under solar minimum conditions. From just past the dawn-dusk terminator line the model is replaced by the lines of a Mach cone. Comparisons of other solar minimum bow shock fits show that the greater accuracy of VEX and the statistical factor makes a significant difference in the positioning of the bow shock fit.

[46] Comparisons between bow shock positions have shown that, in a similar way to the solar cycle making a difference to the bow shock position between maximum and minimum, the solar activity at minimum also affects the bow shock position. Considering the small number of data points, further fits are required in future solar minima to confirm and quantize the linear relations.

[47] The comparisons of the F10.7 index and the Lyman alpha flux show that EUV radiation is more important in this change of position. The data periods are still worthy of further investigation, as it may be that other factors (such as solar transient events) have caused an expansion of the bow shock outwards at these times.

[48] Acknowledgment. Thomas Cravens thanks the reviewers for their assistance in evaluating this paper.

\section{References}

Argo, H. V., et al. (1967), Observations of Solar Wind Plasma Changes across the Bow Shock, J. Geophys. Res., 72, 1989-1994, doi:10.1029/ JZ072i007p01989. 
Barabash, S., et al. (2007), The Analyser of Space Plasmas and Energetic Atoms (ASPERA-4) for the Venus Express mission, Planet. Space Sci., 55, 1772-1792, doi:10.1016/j.pss.2007.01.014.

Formisano, V. (1979), Orientation and shape of the Earth's bow shock in three dimensions, Planet. Space Sci., 27(9), 1151-1161, doi:10.1016/ 0032-0633(79)90135-1.

Gibson, S. E., J. U. Kozyra, G. de Toma, B. A. Emery, T. Onsager, and B. J. Thompson (2009), If the Sun is so quiet, why is the Earth ringing? A comparison of two solar minimum intervals, J. Geophys. Res., 114 , A09105, doi:10.1029/2009JA014342.

Luhmann, J. G., et al. (1986), The solar wind interaction with Venus, Space Sci. Rev., 44, 241-306, doi:10.1007/BF00200818.

Martinecz, C., et al. (2008), Location of the bow shock and ion composition boundaries at Venus-initial determinations from Venus Express ASPERA-4, Planet. Space Sci., 56, 780-784, doi:10.1016/j.pss. 2007.07.007.

Masters, A., N. Achilleos, M. K. Dougherty, J. A. Slavin, G. B Hospodarsky, C. S. Arridge, and A. J. Coates (2008), An empirical model of Saturn's bow shock: Cassini observations of shock location and shape, J. Geophys. Res., 113, A10210, doi:10.1029/2008JA013276.

Phillips, J. L., and D. J. McComas (1991), The magnetosheath and magnetotail of Venus, Space Sci. Rev., 55(1-4), 1-80.

Phillips, J. L., J. G. Luhmann, and C. T. Russell (1985), Dependence of Venus ionopause altitude and ionospheric magnetic field on solar wind dynamic pressure, Adv. Space Res., 5(9), 173-176, doi:10.1016/0273$1177(85) 90286-8$

Rasool, S. I. (1968), Loss of water from Venus, J. Atmos. Sci., 25(4), 663-664, doi:10.1175/1520-0469(1968)025<0663:LOWFV>2.0.CO;2.

Russell, C. T., and O. Vaisberg (1983), The interaction of the solar wind with Venus, in Venus, edited by D. M. Hunten et al., pp. 873-940, Univ. of Ariz. Press, Tucson, Ariz.

Russell, C. T., E. Chou, J. G. Luhmann, P. Gazis, L. H. Brace, and W. R. Hoegy (1988), Solar and interplanetary control of the location of the Venus bow shock, J. Geophys. Res., 93, 5461-5469, doi:10.1029/ JA093iA06p05461.

Slavin, J., R. Holzer, J. Spreiter, and S. Stahara (1984), Planetary Mach cones-Theory and observation, J. Geophys. Res., 89, 2708-2714, doi:10.1029/JA089iA05p02708.

Trotignon, J. G., et al. (2006), Martian shock and magnetic pile-up boundary positions and shapes determined from the Phobos 2 and Mars Global Surveyor data sets, Planet. Space Sci., doi:10.1016/j.pss.2006.01.003.

Woods, T. N., W. K. Tobiska, G. J. Rottman, and J. R. Worden (2000), Improved solar Lyman alpha irradiance modeling from 1947 through 1999 based on UARS observations, J. Geophys. Res., 105(A12), 27,195-27,215, doi:10.1029/2000JA000051.
Zhang, T., J. G. Luhmann, and C. T. Russell (1990), The solar cycle dependence of the location and shape of the Venus bow shock, J. Geophys. Res., 95, 14,961-14,967, doi:10.1029/JA095iA09p14961.

Zhang, T., et al. (2008), Initial Venus Express magnetic field observations of the Venus bow shock location at solar minimum, Planet. Space Sci., 56, 785-789, doi:10.1016/j.pss.2007.09.012.

H. Andersson, S. Barabash, Y. Futaana, A. Grigoriev, M. Holmström, R. Lundin, and M. Yamauchi, Swedish Institute of Space Physics, S-98128 Kiruna, Sweden.

K. Asamura, Institute of Space and Astronautical Science, 3-1-1 Yoshinodai, Sagamihara 229-8510, Japan.

W. Baumjohann, H. Lammer, and T. L. Zhang, Space Research Institute, Austrian Academy of Sciences, A-8042 Graz, Austria.

P. Bochsler and P. Wurz, Physikalisches Institut, University of Bern, CH-3012 Bern, Switzerland.

P. Brandt and E. Roelof, Johns Hopkins University Applied Physics Laboratory, Laurel, MD 20723-6099, USA.

R. Cerulli-Irelli, M. Maggi, M. Milillo, A. Mura, and S. Orsini, Instituto di Fisica dello Spazio Interplanetario, I-00133 Rome, Italy.

A. J. Coates, D. O. Kataria, and D. R. Linder, Mullard Space Science Laboratory, UCL, Holmbury St. Mary, Dorking, Surrey RH5 6NT, UK.

C. C. Curtis and K. C. Hsieh, Department of Physics, University of Arizona, Tucson, AZ 85721, USA.

A. Federov, C. Mazelle, J. A. Sauvaud, and J. J. Thocaven, Centre d'Étude Spatiale des Rayonnements, BP-44346, F-31028 Toulouse, France.

R. A. Frahm and J. R. Sharber, Southwest Research Institute, San Antonio, TX 78228-0510, USA.

M. Fränz, Max-Planck-Institut für Sonnensystemforschung, MaxPlanck-Str. 2, D-37191 Katlenburg-Lindau, Germany.

M. Grande, G. Guymer, B. Pintér, and I. Whittaker, Aberystwyth University, Penglais, Aberystwyth, Ceredigion SY23 3BZ, UK. (icw06@ aber.ac.uk)

E. Kallio, H. E. J. Koskinen, P. Riihelä, and W. Schmidt, Finnish Meteorological Institute, Box 503, FIN-00101 Helsinki, Finland.

J. Kozyra, Space Physics Research Laboratory, University of Michigan, Ann Arbor, MI 48109-2143, USA.

S. McKenna-Lawlor, Space Technology Ireland, National University of Ireland, Maynooth, Co. Kildare, Ireland.

C. T. Russell, IGPP, University of California, Los Angeles, CA 90095 , 